\title{
Emotional context, maternal behavior and emotion regulation
}

\author{
Lisa Roque, Manuela Veríssimo* \\ UIPCDE, Unidade de Investigação em Psicologia Cognitiva, do Desenvolvimento e da Educação, ISPA - Instituto Universitário, Rua Jardim do Tabaco, 34, 1149 \\ - 041 Lisboa, Portugal
}

\section{A R T I C L E I N F O}

\section{Article history:}

Received 15 January 2011

Received in revised form 3 May 2011

Accepted 21 June 2011

\section{Keywords:}

Emotion regulation

Positive and negative affect-contexts

Maternal involvement

Expressiveness

Intensity

\begin{abstract}
A B S T R A C T
This study investigated the importance of emotion-eliciting context (positive and negative) and mother's behaviors (constrained and involved) on toddlers' emotion regulation behavioral strategies, emotional expressiveness and intensity, during three episodes eliciting fear, frustration/anger and positive affect. Fifty-five children between 18 and 26 months of age and their mothers participated in the study. Toddlers' regulatory strategies varied as function of emotion-eliciting context (children exhibited behavioral strategies more frequently during positive affect and frustration/anger episodes and less frequently during fear episodes) and maternal involvement. Toddlers' expression of emotion varied as function of emotion-eliciting context (children exhibited more emotional expressions, both negative and positive during fear and frustration/anger episodes compared to positive affect episodes). Toddlers' expression of emotion was not strongly related to maternal involvement, however, the intensity of emotional expression was related to the interaction of context and maternal involvement.
\end{abstract}

(c) 2011 Elsevier Inc. All rights reserved.

Rothbart and Sheese (2007) argue that emotion regulation should be construed as a biopsychosocial behavioral system responsible for the modulation of emotional reactions, including its inhibition, activation or graded modulation. This system involves changes in latency, rise times, magnitudes and durations of responses in behavior, experience and physiology, depending on an individual's goals (Gross \& Thompson, 2007; Thompson \& Meyer, 2007). According to the functionalist perspective (Campos, Mumme, Kermoian, \& Campos, 1994) emotion regulation is concerned with relations between emotion(s) and the person's immediate or long-term objectives. When emotions are experienced in a flexible and controlled manner (i.e., regulated), they are useful in mobilizing the individual's behavioral resources in the service of goal attainment, including establishing inter-personal relationships, engaging in pro-social initiatives, self-assertiveness, etc., according to the social and cultural demands of the context and the individual's objectives. In principle, advances in emotion regulation skills promote adaptive behaviors and appropriate and flexible responses to the initiations of others (Denham, 1998; Fox, 1994). Failure to attain age-appropriate emotion regulatory skills (i.e., dysregulation) is reflected by the use of developmentally primitive regulatory strategies and the inability to make decisions regarding appropriate conduct across diverse social contexts (Denham, 1998; Fox, 1994). Furthermore, emotional dysregulation has been associated with psychopathological symptoms and/or deviant developmental trajectories in childhood and adolescence (Cole, Michael, \& Teti, 1994; Maughan \& Cicchetti, 2002).

\section{Regulation of positive and negative emotions (fear and frustration/anger)}

During fear contexts, strategies such as withdrawal (Buss \& Goldsmith, 1998) avoidance and fussing to mother (Diener \& Mangelsdorf, 1999a), were associated with a decrease in the intensity of children's fear expressions.On the other hand, when

\footnotetext{
* Corresponding author.

E-mail address: mveriss@ispa.pt (M. Veríssimo).
} 
children used approach and interaction strategies with the stimulus (Buss \& Goldsmith, 1998) or played with it (Diener \& Mangelsdorf, 1999a), there was no change in the intensity of fear expressions.

In the study of anger regulation, decreases in anger were associated with three strategies in children: shifting attention away from sources of frustration, passive waiting and seeking information; while focusing on the frustrating event was associated with increases in anger intensity (Gilliom, Shaw, Beck, Schonberg, \& Lukon, 2002).

When comparing strategies used in the regulation of fear and frustration/anger episodes, research suggests that children tend to use more regulatory strategies during frustration/anger contexts, than during fear episodes, probably due to the fact that the frustration object is unattainable and children try more strategies in a repeated way in order to get it back, whereas this is unnecessary during fear episodes (Diener \& Mangelsdorf, 1999a).

A review of literature points to a lack of attention to positive affect in the study of emotion regulation. To our knowledge, few studies (Beauregard, Levesque, \& John, 2001; Giuliani, McRae, \& Gross, 2008; Kim \& Hamann, 2007) have analysed the regulation of positive affect, and none of these included children. Research on the regulation of positive affect is essential, particularly, the study of strategies that maintain and increase the experiences of positive emotions, since cultivating them may be particularly important for building resilience to stressful events (Tugade \& Fredrickson, 2007). The regulation of positive affect also includes the modulation of high intensity positive affect in contexts where it is not appropriate. Intense positive experiences can have undesirable consequences, such as greater arousal or physiological reactivity, which may impair the benefit of positive emotions. Therefore, although intense positive experiences are desirable at the time they are experienced, they may not be related to long-term well being (Diener, Sandvik, \& Pavot, 1991). The regulation of positive and negative affect is equally important, particularly, if it contributes to the attainment of one's immediate or long term goals (Campos et al., 1994). However, no studies have been done to show if there are any differences in the number of regulatory strategies exhibited during positive affect episodes, compared to negative affect ones. According to learning and operant conditioning theories, behaviors are strengthened by the consequence of experiencing a positive condition, but also by the consequence of stopping or avoiding a negative one (Malott \& Trojan, 2008). This also suggests that there might be no differences in the frequency of regulatory strategies exhibited during positive and negative emotion-eliciting contexts.

\section{The role of mother's involvement}

Maternal involvement is extremely important in regulating the child's physiological and emotional arousal states, either by: (1) providing the means for the child's physical survival and well being (food, shelter, clothing, and physical soothing); or (2) through more complex interactions (caregiving styles or explicit training, like discipline, modeling, and reinforcement), which teach the child how to manage stress, frustration and how to control impulses (Calkins, 1994). This process of external regulation is progressively internalized by the child and becomes a source of self-regulation, particularly, when sensorimotor behavior and locomotion are developed, and social referencing skills are acquired. These new developmental processes allow the child to regulate physical and emotional proximity to the caregiver and people in general, and to facilitate access to new sources of information (Kopp, 1989).

Children between 18 and 24 months, use different behavioral strategies as a function of maternal involvement, in situations of fear and anger (Diener \& Mangelsdorf, 1999a). For example, they are more likely to behave in ways that distract them from a goal-object during delay situations when an adult is available and involved (Grolnick, Bridges, \& Connell, 1996). They also engage, social reference their mothers, play with the stimulus, and express more positive than negative affect during mother involved periods, as compared with periods during which maternal behavior is constrained by experimenter instruction (Diener \& Mangelsdorf, 1999a).

Research also suggests that maternal involvement and the quality of the attachment relationship influence children's emotional expressiveness. Insecure children, tend to either minimize or exaggerate emotion expressiveness (Cassidy, 1994; Bretherton, 1990). On the other hand, secure children are characterized by an open, direct, and active expression of affect to the mother in case of distress and the pair often exchange expressions of joy during positive affect episodes that serve to maintain interest in their relationship (Bowlby, 1969/1982; Bretherton, 1990).

\section{Aims of the study}

The main goal of this study was to explore the influences of the emotion-eliciting context (positive and negative affect) and maternal behaviors (constrained or involved) on toddlers' emotional expressiveness, intensity, and the exhibition of behaviors that might regulate either expressiveness or intensity. Observations were conducted at children's homes and not at the laboratory, which may provide important insight to the understanding of children's emotion regulation during daily-life events, outside more controlled settings. Our approach to emotion regulation focuses on the study of frequency of strategies and emotional expressions as function of maternal involvement, and not on the study of changes in the intensity of emotional expressions (Diener \& Mangelsdorf, 1999a). This approach is supported by the work of Diener et al. (1991) which argues that happiness researchers should assess primarily the relative frequency of positive versus negative emotional experience. Two reasons are presented by the authors: (1) the relative frequency of positive emotions can be more accurately and validly measured; (2) frequent positive affect is both necessary and sufficient to produce the state we call happiness, whereas intense positive experience is not, since it can have undesirable features. 
First, we explored relations between (potentially) regulatory behaviors, maternal involvement and emotion-eliciting contexts, either positive or negative (fear, frustration/anger). We hypothesized that: (a) toddlers' use of regulatory behaviors will vary as function of the emotion-eliciting context and that children will show these behaviors more frequently during frustration/anger episodes, than during fear contexts (Diener \& Mangelsdorf, 1999a). No differences are expected between positive affect episodes and negative affect ones, either fear or anger. This can be explained by the fact that emotion regulation may be related either with the inhibition of negative affect, or the maintenance of positive emotions, according to one's goals (Campos et al., 1994; Thompson, 1994) and that both positive and negative experiences strengthen and reinforce behavior (Malott \& Trojan, 2008); (b) Toddlers' regulatory behaviors will vary as function of maternal involvement, specifically that children will be more likely to display regulatory behaviors during mother involved periods, compared to mother constrained ones during fear episodes (as a way to get comfort) and more likely to display regulatory behaviors during mother constrained periods, than during mother involved ones, in the frustration anger episodes (as a way to get the toy back and play). During positive affect episodes, we expected that children will show no differences in the frequency of regulatory behaviors used in mother constrained and involved periods, given that in both moments the desired object is always present and represents a source of pleasure, even if the mother is emotionally unavailable.

The second aim was to examine the links between toddlers' emotional expressiveness, maternal behavior, and context. We expected that: (a) emotional expression would show no differences in its frequency as function of emotion-eliciting context. Expressiveness may be used by children as a way to signal their mothers' about their needs and goals, that is, to play in positive affect contexts or to be soothed in negative affect contexts (Campos et al., 1994); (b) emotional expressiveness will vary as result of maternal involvement, and children will exhibit emotional expressions more frequently, particularly positive affect ones, during mother involved periods, than during mother constrained ones. This is as a result of the social interactive aspects of emotion regulation, developed at the end of the first year (Kopp, 1989; Cassidy, 1994; Bretherton, 1990).

The third goal was to explore toddlers' emotional intensity, during positive and negative affect contexts and mother constrained and involved periods. We hypothesized that: (a) emotional intensity will not vary as a function of negative or positive affect contexts, because emotion regulation may involve the inhibition or the maintenance and enhancement of emotion, either positive or negative, according to the demands of the situation (Gross \& Thompson, 2007; Thompson, 1994); (b) emotional intensity will vary with maternal involvement. Children will exhibit higher emotional intensity during mother constrained periods, than during mother involved ones, as a way to call mothers' attention to their needs, either to diminish distress or to play (Campos et al., 1994; Kopp, 1989).

\section{Method}

\subsection{Participants}

Fifty-five mother/child dyads (27 boys and 28 girls), all Caucasian, from bi-parental families participated in the study. Children were between 18 and 26 months of age $(M=21.35 ;$ S.D. $=1.91)$. Twenty-seven were firstborn and twenty-eight had siblings. They started attending day-care centers between the age of 6 and 24 months $(M=7.53 ; S . D .=4.81)$ and spent $7-11 \mathrm{~h}(M=6.96 ;$ S.D. $=2.64)$ in day-care each weekday. Mothers' age ranged from 25 to 43 years $(M=33.64 ; S . D .=4.10)$ and fathers' age from 26 to 55 years old $(M=35.71 ; S . D .=5.73)$. Mothers' level of education ranged from 5 to 19 years $(M=14.87$; S.D. $=3.38)$ and fathers' from 4 to 19 years $(M=13.71 ;$ S.D. $=3.60)$. Participants represented a range of socioeconomic status backgrounds, as reflected by parental education and were recruited from public and private daycare centers.

\subsection{Measures}

\subsubsection{Emotion regulation paradigm: fear, positive affect, frustration/anger}

The emotion regulation paradigm (Diener \& Mangelsdorf, 1999a), measures behavioral strategies, emotional expressiveness and intensity exhibited by children during three episodes: positive affect, fear and frustration/anger, elicited by presenting the children three different toys. Each episode lasted for 6 min and had two distinct moments of 3 min each: (1) mother constrained period (mothers were instructed to refrain from initiating interaction with their children, if their children made bids for attention, mothers were instructed to respond to them with brief statements about the stimuli presented in each episode: "It's the dinosaur/piano/bear"); (2) mother involved period (mothers were instructed to be at ease with the child and the toy. Free behavior was allowed, whatever they felt it was appropriate, according to their sensitivity). During mother constrained period, if the children showed $30 \mathrm{~s}$ of sustained high-intensity distress, mothers were instructed to become involved. If this situation happened during the mother involved periods, the episode was terminated. All episodes were videotaped.

4.2.1.1. Emotional stimuli. All stimuli used in the present study were previously tested in a pilot study, which showed a varying emotional intensity in most children. During the frustration/anger episode, we presented children with a movable box with wheels, shaped in the form of a yellow bear, which contained colored lego pieces inside. After the experimenter felt that the child was involved with the toy ( 2 min on average), he took the toy away firmly and placed it out of reach but within the child's sight. During the constrained period, mothers' were instructed not to give back the toy to their children. During 
the mother involved period, when free behavior was allowed, all the mothers decided to give back the toy to their children, immediately after the beginning of this period. During fear episodes, a dinosaur with elements of novelty, unpredictability and intrusiveness was used to elicit fear. During the positive affect episode, children were given a toy piano that played music and created musical rhythms. Similar procedures for fear and frustration/anger episodes (but with different stimuli) were used in other studies (Buss \& Goldsmith, 1998; Diener \& Mangelsdorf, 1999a; Grolnick et al., 1996).

4.2.1.2. Children behavioral strategies. Toddlers' behavioral strategies were divided into four domains (see Diener \& Mangelsdorf, 1999a, 1999b): (1) mother-related strategies (proximity/contact seeking to mother; directing mother; fuss to mother; help seeking; information seeking; social referencing/looks to mother; engagement of mother); (2) disengagement of attention strategies (passive disengagement; distraction towards other object or person/active disengagement; leave taking; avoidance); (3) dealing with the stimulus strategies (playing/exploring; resistance/control; labeling; problem solving; proximity to stimulus); (4) redirection of action strategies (tension release; self-soothing). During the course of our study, another set of behaviors was observed, besides the ones proposed by Diener and Mangelsdorf (1999b). This one was coded under the name of "stranger", because it was characterized by behaviors directed at the strangers (experimenters) in the room during the sessions and it was placed in the "redirection of action strategies" domain.

Children's behavioral strategies were coded dichotomously on an occurrence/non occurrence way, each $15 \mathrm{~s}$ intervals ( 1 - occurrence; 0 - non occurrence). Each 3-min period had $1215 \mathrm{~s}$ intervals. The results for each strategy were summed for a total score. The possible range for each behavior was $0-12$, for each 3 -min period. If an episode was terminated because of child distress, scores were prorated on the basis of the number of intervals completed, by dividing the sums of the scores by the number of intervals completed and multiplying 12 (the total number of intervals possible) (Diener \& Mangelsdorf, 1999a).

4.2.1.3. Emotional expression. The predominant emotion the child expressed in each 15-s interval was also coded (Diener \& Mangelsdorf, 1999a). Fear was scored when the child expressed at least one of these facial features: eyebrows raised or drawn together; eyes wide; mouth open, corners straight back. Positive affect was scored when the child smiled or produced a positive vocalization (laugh). Anger was coded when the child showed at least one of the following: brows pulled back down or together; raised cheeks; straight or angular mouth or tight lips. A "neutral" score was given when the child did not express any of the target emotions and these scores were not retained for analysis. If the child expressed more than one emotion during the 15-s interval, the most intensive one was coded as the predominant one.

4.2.1.4. Emotional intensity. The intensity of the emotions expressed by the child was scored in a scale of $1-3$ points (1 - low intensity; 2 - medium intensity; 3 - high intensity) for each 15-s interval. High intensity emotions could be expressed by facial affect, body postures, gestures and movements or full intensity vocalizations (e.g., laughter for positive affect; crying or screaming for negative affect). Low intensity affect seemed mild and would be more ambiguous than high intensity one (Diener \& Mangelsdorf, 1999b).

Independent coders, blind to the aims of the study coded the three episodes. Inter-rater reliability was calculated using Cohen's Kappas (fear =.73; positive affect $=.84$; frustration/anger $=.70$ ). This coding system is similar to those used in other studies of children coping strategies (Buss \& Goldsmith, 1998; Calkins \& Johnson, 1998; Diener \& Mangelsdorf, 1999b; Nachmias, Gunnar, Mangelsdorf, Parritz, \& Buss, 1996; Parritz, 1996).

\subsection{Procedures}

The emotion regulation episodes were videotaped at the children's home in different days, in order to avoid any emotional contamination from one episode to the other. They all started at the same time (18:30). The time chosen to start the experiments was late afternoon, because $94 \%$ of the mothers worked outside the home and finish their shift around 17:00. The three episodes were counter-balanced across subjects in order to control any order effect over the results.

\section{Results}

\subsection{Preliminary analyses}

First, we tested if the emotional manipulations were effective and if the target emotion was expressed more frequently in the correspondent episode, than the other emotions in a significant way. A repeated measures MANOVA was conducted. When the results were significant, relevant differences were tested with planned contrast estimates analyses. Two withineffects levels were used: emotional expression (fear, positive affect and frustration/anger facial expressions) and episode (fear, positive affect, and frustration/anger). Child gender was used as between-effect or independent variable. The analysis revealed significant main effects for episode $(F(2,106)=37.94, p<.001)$ and emotional expression $(F(2,106)=7.35, p<.001)$. A significant interaction between episode and emotional expression was also found $(F(4,212)=80.36, p<.001)$. No gender effects were found.

During fear episodes (see Table 1) children showed fearful expressions significantly more often, than positive affect $(t$ $(53)=2.38, p<.05)$ and more fear than frustration/anger expressions $(t(53)=10.23, p<.001)$. During positive affect episodes, 
Table 1

Means and standard errors for children's emotional expressions, during episodes of fear, positive affect and frustration/anger.

\begin{tabular}{|c|c|c|c|}
\hline Episode & Emotional expression & $M$ & S.E. \\
\hline \multirow[t]{3}{*}{ Fear } & Positive affect expression & 3.91 & 0.58 \\
\hline & Frustration/anger expression & 0.30 & 0.09 \\
\hline & Fear expression & 6.50 & 0.57 \\
\hline \multirow[t]{3}{*}{ Positive affect } & Positive affect expression & 4.93 & 0.52 \\
\hline & Frustration/anger expression & 0.71 & 0.16 \\
\hline & Fear expression & 0.81 & 0.24 \\
\hline \multirow[t]{3}{*}{ Frustration/anger } & Positive affect expression & 2.31 & 0.26 \\
\hline & Frustration/anger expression & 6.13 & 0.31 \\
\hline & Fear expression & 0.00 & 0.00 \\
\hline
\end{tabular}

Table 2

Means and standard errors for children's four types of emotional regulation strategies as function of episode and maternal involvement.

\begin{tabular}{|c|c|c|c|c|c|c|c|c|c|c|c|c|}
\hline \multirow[t]{3}{*}{ Strategy type } & \multicolumn{4}{|l|}{ Fear } & \multicolumn{4}{|c|}{ Positive affect } & \multicolumn{4}{|c|}{ Frustration/anger } \\
\hline & \multicolumn{2}{|c|}{ Constrained } & \multicolumn{2}{|c|}{ Involved } & \multicolumn{2}{|c|}{ Constrained } & \multicolumn{2}{|c|}{ Involved } & \multicolumn{2}{|c|}{ Constrained } & \multicolumn{2}{|c|}{ Involved } \\
\hline & $M$ & S.E. & $M$ & S.E. & $M$ & S.E. & $M$ & S.E. & $M$ & S.E. & $M$ & S.E. \\
\hline \multicolumn{13}{|l|}{ Mother-related } \\
\hline Proximity to mother & 8.74 & 0.58 & 9.62 & 0.47 & 8.08 & 0.51 & 9.60 & 0.46 & 8.55 & 0.55 & 9.59 & 0.38 \\
\hline Directing mom & 0.74 & 0.25 & 0.92 & 0.21 & 0.68 & 0.18 & 0.64 & 0.19 & 3.86 & 0.55 & 3.43 & 0.43 \\
\hline Fussing to mother & 2.02 & 0.34 & 2.18 & 0.36 & 0.24 & 0.09 & 0.33 & 0.17 & 2.53 & 0.53 & 0.68 & 0.26 \\
\hline Helpseeking & 0.11 & 0.07 & 0.03 & 0.02 & 0.05 & 0.03 & 0.07 & 0.03 & 2.86 & 0.51 & 1.84 & 0.34 \\
\hline Information seeking & 0.08 & 0.05 & 0.07 & 0.04 & 0.04 & 0.02 & 0.13 & 0.08 & 0.42 & 0.24 & 0.28 & 0.16 \\
\hline Social referencing & 4.13 & 0.41 & 5.13 & 0.39 & 5.54 & 0.45 & 6.26 & 0.46 & 6.75 & 0.47 & 6.48 & 0.45 \\
\hline Engaging mother & 1.59 & 0.36 & 1.01 & 0.25 & 3.36 & 0.44 & 5.09 & 0.44 & 5.08 & 0.53 & 5.40 & 0.44 \\
\hline \multicolumn{13}{|l|}{ Disengagement of attention } \\
\hline Passive disengagement of attention & 0.80 & 0.21 & 0.67 & 0.23 & 4.33 & 0.41 & 3.21 & 0.38 & 3.79 & 0.43 & 1.28 & 0.28 \\
\hline Distraction & 0.24 & 0.11 & 0.71 & 0.23 & 1.57 & 0.35 & 1.70 & 0.40 & 2.88 & 0.48 & 1.44 & 0.36 \\
\hline Leave taking & 0.07 & 0.04 & 0.09 & 0.05 & 0.00 & 0.00 & 0.22 & 0.14 & 0.45 & 0.18 & 0.24 & 0.12 \\
\hline Avoidance & 2.13 & 0.28 & 3.92 & 0.45 & 0.91 & 0.23 & 1.57 & 0.37 & 2.36 & 0.50 & 1.91 & 0.35 \\
\hline \multicolumn{13}{|l|}{ Dealing with the stimulus } \\
\hline Playing with stimulus & 0.47 & 0.21 & 2.15 & 0.44 & 8.16 & 0.58 & 8.12 & 0.53 & - & - & - & - \\
\hline Resistance/control & 0.04 & 0.03 & 0.16 & 0.07 & 0.00 & 0.00 & 0.09 & 0.07 & - & - & - & - \\
\hline Labeling & 1.41 & 0.31 & 2.19 & 0.35 & 0.27 & 0.16 & 0.11 & 0.06 & 0.25 & 0.15 & 0.20 & 0.16 \\
\hline Problem solving & 0.43 & 0.22 & 1.02 & 0.27 & 0.58 & 0.20 & 1.22 & 0.34 & 0.21 & 0.10 & 0.77 & 0.32 \\
\hline Proximity to stimulus & 3.00 & 0.60 & 7.27 & 0.58 & 9.39 & 0.50 & 10.13 & 0.38 & 1.92 & 0.46 & 8.80 & 0.46 \\
\hline \multicolumn{13}{|l|}{ Re-directed action } \\
\hline Tension release & 1.60 & 0.34 & 1.23 & 0.30 & 0.17 & 0.08 & 0.40 & 0.14 & 1.54 & 0.41 & 0.47 & 0.23 \\
\hline Self-soothing & 6.19 & 0.71 & 5.29 & 0.70 & 4.29 & 0.70 & 2.85 & 0.60 & 4.48 & 0.69 & 3.51 & 0.67 \\
\hline Stranger & 0.50 & 0.18 & 0.59 & 0.16 & 4.02 & 0.45 & 3.03 & 0.43 & 0.63 & 0.18 & 0.25 & 0.10 \\
\hline Total & 1.80 & 0.08 & 2.33 & 0.07 & 2.72 & 0.06 & 2.88 & 0.07 & 2.56 & 0.12 & 2.81 & 0.09 \\
\hline
\end{tabular}

they showed positive affect expressions significantly more often, than fearful ones $(t(53)=6.19, p<.001)$ and more positive affect than frustration/anger expressions $(t(53)=7.34, p<.001)$. Finally, during the frustration/anger episodes children expressed frustration/anger faces significantly more often, than positive affect ones $(t(53)=7.98, p<.001)$ and more frustration/anger expressions than fearful ones $(t(53)=19.48, p<.001)$.

\subsection{Main effects of emotion-eliciting context and mothers' involvement on toddlers' behavioral strategies}

A repeated measures MANOVA was conducted and three within-subject levels were used: episode (fear, positive affect, and frustration/anger); maternal condition (constrained and involved) and the 19 strategies. Child gender was used as between-effects factor. To reduce the family-wise type I error rate, only the analyses significant at the $p<.01$ level were discussed. Significant main effects were found for emotion regulation strategies $(F(18,954)=165.13, p<.001)$; episode $(F$ $(2,106)=36.19, p<.001)$ and maternal condition $(F(1,53)=26.87, p<.001)$.

During fear episodes (see Table 2) planned contrast estimates analyses revealed that children exhibited playing with stimulus strategies significantly more often during mother involved periods, than during mother constrained ones $(t(53)=4.04$, $p<.001$ ). They also looked for proximity to the stimulus significantly more often during mother involved periods, than during constrained ones $(t(53)=7.78, p<.001$ ). In total, in fear episodes (see Table 2 ) children exhibited behavioral strategies significantly more often during mother involved periods, than during the mother constrained ones $(t(53)=5.76, p<.001)$.

During positive affect episodes (see Table 2 ) children engaged their mothers significantly more often during mother involved periods, than during constrained ones $(t(53)=3.68, p<.001)$. They passively disengaged their attention significantly more often during mother constrained periods, than during involved ones $(t(53)=2.96, p<.001)$. Children self-soothed themselves significantly more often when their mothers where constrained, than when their mothers where involved $(t$ 
Table 3

Means and standard errors for children's emotional regulation strategies as function of episode and maternal involvement.

\begin{tabular}{|c|c|c|c|c|c|c|}
\hline \multirow[t]{2}{*}{ Episode } & \multicolumn{2}{|c|}{ Constrained } & \multicolumn{2}{|c|}{ Involved } & \multicolumn{2}{|l|}{ Total } \\
\hline & $M$ & S.E. & $M$ & S.E. & $M$ & S.E. \\
\hline Fear & 1.80 & 0.08 & 2.33 & 0.07 & 2.07 & 0.06 \\
\hline Positive affect & 2.72 & 0.06 & 2.88 & 0.07 & 2.80 & 0.06 \\
\hline Frustration/anger & 2.56 & 0.12 & 2.81 & 0.09 & 2.68 & 0.09 \\
\hline
\end{tabular}

$(53)=3.10, p<.001)$. Finally, children directed behaviors towards the strangers significantly more often when their mothers' behavior was constrained, than when it was involved $(t(53)=2.72, p<.001)$.

During frustration/anger episodes (see Table 2) children exhibited fussing to mother behaviors significantly more often during mother constrained periods, than during mother involved ones $(t(53)=4.05, p<.001)$. They engaged in passive disengagement of attention strategies significantly more often when their mothers were constrained, than when their mothers where involved $(t(53)=5.33, p<.001)$. Children distracted themselves from the stimulus significantly more often during mother constrained periods, than during mother involved ones $(t(53)=2.73, p<.001)$. They exhibited proximity to stimulus behaviors significantly more often when the mothers were involved, than when they were constrained $(t(53)=11.13$, $p<.001)$. Children also released tension significantly more often during the mother constrained periods, than during mother involved ones $(t(53)=2.61, p<.01)$. Playing with stimulus and resistance/control strategies were taken out of this analysis because during the mother constrained period, the toy was taken away from the child to a place where they could see it, but could not touch it.

Independently of mothers' involvement, children showed behavioral strategies significantly more often during positive affect episodes, followed by frustration/anger and by fear episodes (see Table 3). However, planned contrast estimates analyses revealed that significant differences only occurred between fear and positive affect episodes $(t(53)=10.47, p<.001)$ and between fear and frustration/anger episodes $(t(53)=6.10, p<.01)$, but not between positive affect and frustration/anger episodes.

\subsection{Interactions between emotion-eliciting context and maternal involvement on toddlers' emotion regulation strategies}

A significant interaction strategies $\times$ episode $\times$ maternal condition $(F(36,1908)=9.55, p<.001)$ was found .

During mother constrained periods (see Table 3), children exhibited behavioral strategies significantly more often during positive affect episodes, followed by frustration/anger and finally by fear episodes. However, planned contrast estimates analyses revealed that significant differences only occurred between fear and positive affect episodes $(t(53)=9.89, p<.001)$ and between fear and frustration/anger episodes $(t(53)=5.90, p<.01)$, but not between positive affect and frustration/anger episodes. On the other hand, during mother involved periods (see Table 3 ) children exhibited strategies significantly more often during positive affect episodes, followed by frustration/anger and finally by fear episodes. However, significant differences only occurred between fear and positive affect episodes $(t(53)=3.76, p<.001)$ and fear and frustration/anger episodes $(t(53)=2.55, p<.01)$, but not between positive affect and frustration/anger episodes.

\subsection{Main effects of emotion-eliciting context and mothers' involvement on toddlers' emotional expressiveness}

A repeated measures MANOVA was conducted in order to examine any significant differences in children's emotional expressiveness. We used three within-effects levels: emotional expressions (fear, positive affect and frustration/anger); episode and maternal condition. Child gender was used as a between-effect factor. The analysis revealed significant main effects for emotional facial expressions $(F(2,106)=7.35, p<.001)$ and episode $(F(2,106)=37.94, p<.001)$. No child gender effects were observed.

Independently of mothers' behavior (see Table 4) children showed expressions (positive and negative) significantly more often during fear episodes, than during positive affect ones $(t(53)=8.69, p<.001)$ and frustration/anger episodes $(t(53)=5.23$, $p<.001)$. They also exhibited expressions significantly more often during frustration/anger episodes, than during positive affect ones $(t(53)=3.69, p<.001)$.

\subsection{Interactions between emotion-eliciting context and maternal involvement on toddlers' emotional expressiveness}

Significant interactions between emotional expression $\times$ episode $(F(4,212)=80.36, p<.001)$; emotional expression $\times$ maternal condition $(F(2,106)=34.24, p<.001)$ and emotional expression $\times$ episode $\times$ maternal condition $(F(4$, $212)=27.76, p<.001)$ were found.

During positive affect episodes (see Table 4) children expressed positive affect significantly more often during mother involved periods, than during mother constrained ones $(t(53)=3.62, p<.001)$. They also showed significantly more often frustration/anger facial expressions when their mothers' behavior was involved, than when it was constrained $(t(53)=2.27$, $p<.001)$. Children expressed fearful facial expressions significantly more often during mother constrained episodes, than during mother involved ones $(t(53)=3.11, p<.001)$. 
Table 4

Means and standard errors for children's emotional expressiveness as function of emotion-eliciting context and maternal involvement.

\begin{tabular}{|c|c|c|c|c|c|c|c|}
\hline \multirow[t]{2}{*}{ Episode } & \multirow[t]{2}{*}{ Emotional expression } & \multicolumn{2}{|c|}{ Constrained } & \multicolumn{2}{|c|}{ Involved } & \multicolumn{2}{|c|}{ Total } \\
\hline & & $M$ & S.E. & $M$ & S.E. & $M$ & S.E. \\
\hline \multirow[t]{4}{*}{ Fear } & Positive affect expression & 3.29 & 0.63 & 4.52 & 0.65 & 3.91 & 0.58 \\
\hline & Frustration/anger expression & 0.20 & 0.10 & 0.39 & 0.16 & 0.30 & 0.09 \\
\hline & Fear expression & 6.67 & 0.70 & 6.36 & 0.63 & 6.50 & 0.57 \\
\hline & Total & 3.39 & 0.17 & 3.76 & 0.11 & 3.57 & 0.11 \\
\hline \multirow[t]{4}{*}{ Positive affect } & Positive affect expression & 4.07 & 0.58 & 5.82 & 0.56 & 4.93 & 0.52 \\
\hline & Frustration/anger expression & 0.33 & 0.12 & 1.09 & 0.30 & 0.71 & 0.16 \\
\hline & Fear expression & 1.29 & 0.36 & 0.35 & 0.19 & 0.81 & 0.24 \\
\hline & Total & 1.89 & 0.18 & 2.42 & 0.17 & 2.16 & 0.15 \\
\hline \multirow[t]{4}{*}{ Frustration/anger } & Positive affect expression & 0.86 & 0.26 & 3.75 & 0.46 & 2.31 & 0.26 \\
\hline & Frustration/anger expression & 9.03 & 0.47 & 3.22 & 0.42 & 6.13 & 0.31 \\
\hline & Fear expression & 0.00 & 0.00 & 0.00 & 0.00 & 0.00 & 0.00 \\
\hline & Total & 3.29 & 0.13 & 2.32 & 0.15 & 2.81 & 0.11 \\
\hline
\end{tabular}

Table 5

Means and standard errors for children's emotional intensity as function of emotion-eliciting context and maternal involvement.

\begin{tabular}{|c|c|c|c|c|c|c|}
\hline \multirow[t]{2}{*}{ Episode } & \multicolumn{2}{|c|}{ Constrained } & \multicolumn{2}{|c|}{ Involved } & \multicolumn{2}{|l|}{ Total } \\
\hline & $M$ & S.E. & $M$ & S.E. & $M$ & S.E. \\
\hline Fear & 18.26 & 0.85 & 18.90 & 0.65 & 18.58 & 0.66 \\
\hline Positive affect & 19.68 & 0.74 & 22.38 & 0.66 & 21.03 & 0.61 \\
\hline Frustration/anger & 21.04 & 0.10 & 18.23 & 0.66 & 19.64 & 0.71 \\
\hline
\end{tabular}

During frustration/anger episodes (see Table 4) children expressed significantly more often, positive affect during mother involved periods, than during mother constrained ones $(t(53)=5.34, p<.001)$. They also showed frustration/anger facial expressions significantly more often during constrained periods, than during involved ones $(t(53)=9.16, p<.001)$.

No significant differences $(p<.01)$ between constrained and involved periods were found during fear episodes.

In mother constrained periods (see Table 4 ) during fear episodes, children showed fearful expressions significantly more often, than positive affect $(t(53)=2.72, p<.001)$ or frustration/anger ones $(t(53)=8.79, p<.001)$. During positive affect episodes (see Table 4) they showed positive affect expressions significantly more often, than fear $(t(53)=3.50, p<.001)$ or frustration/anger ones $(t(53)=6.08, p<.001)$. During frustration/anger episodes (see Table 4$)$ they expressed frustration/anger faces significantly more often, than positive affect $(t(53)=12.37, p<.001)$ or fearful ones $(t(54)=19.02, p<.001)$.

In mother involved periods (see Table 4) during fear episodes, children showed fearful expressions significantly more often, than frustration/anger ones $(t(53)=8.97, p<.001)$. No significant differences between fear and positive affect expressions were found. During positive affect episodes (see Table 4 ) children showed positive affect expressions significantly more often, than frustration/anger $(t(53)=6.62, p<.001)$ or fearful ones $(t(53)=8.43, p<.001)$. During frustration/anger episodes (see Table 4 ) they expressed frustration/anger faces significantly more often, than fearful ones $(t(53)=7.78, p<.001)$. No significant differences were found between frustration/anger and positive affect expressions.

During mother constrained periods, children exhibited expressions either positive or negative (total of expressions per episode) significantly more often during fear episodes, than during positive affect ones $(t(53)=6.79, p<.001)$. They also exhibited expressions significantly more often during frustration/anger episodes, than during positive affect ones $(t(53)=6.04$, $p<.001$ ). No significant differences were found between fear and frustration/anger episodes.

During mother involved periods (see Table 4) children also exhibited expressions (positive or negative), significantly more often during fear episodes, than during positive affect $(t(53)=7.14, p<.001)$ or frustration/anger episodes $(t(53)=7.35$, $p<.001)$. No significant differences were found between positive affect and frustration/anger episodes.

\subsection{Main effects of emotion-eliciting context and mothers' involvement on toddlers' emotional intensity}

When it comes to emotional intensity experienced by children, a repeated measures MANOVA was undertaken. Two within-effects levels were used: episode and maternal condition. Child gender was used as between-effects factor. No main effects were found for episode, maternal condition or child gender.

\subsection{Interactions between emotional eliciting context and maternal involvement on toddlers' emotional intensity}

A significant interaction between episode $\times$ maternal condition was found for toddlers' emotional intensity $(F(2$, 106) $=12.41, p<.001)$.

During fear episodes, no significant differences between maternal constrained and involved periods were found (see Table 5). During positive affect episodes (see Table 5) children exhibited significantly higher emotional intensity during mother involved periods, than during mother constrained ones $(t(53)=4.03, p<.001)$. During frustration/anger episodes 
(see Table 5) children displayed significantly higher emotional intensity when mothers were constrained, than when they were involved $(t(53)=3.01, p<.001)$.

During mother involved periods (see Table 5 ) children showed significantly higher emotional intensity during positive affect episodes, than during fear $(t(53)=3.72, p<.001)$ and frustration/anger ones, $(t(53)=4.97, p<.001)$. No significant differences were found between fear and frustration/anger episodes.

During mother constrained periods no significant differences $p<.01$ were found between episodes.

In total, independently of the mothers' behavior (see Table 5) children exhibited significantly higher emotional intensity during positive affect episodes, than during fear episodes $(t(53)=2.61, p<.01)$. No significant differences were found between positive affect and frustration/anger episodes or between fear and frustration/anger episodes.

\section{Discussion}

Toddlers' behavioral regulatory strategies varied as function of emotion-eliciting episodes. In particular, children used more often strategies during positive affect and frustration/anger episodes and fewer during fear episodes, partially confirming our first hypotheses (Diener \& Mangelsdorf, 1999a). In both positive affect and frustration/anger episodes, the stimuli were desirable objects to play, a piano with musical sounds and legos, respectively. It may have been that the desire to play with the stimulus during the positive affect episodes and the motivation to obtain the object during the frustration/anger ones, made children use more strategies in order to accomplish their immediate goals and self-regulate (Campos et al., 1994). We did not expected to find differences between the frequency of strategies used during positive affect episodes and negative affect ones, however, this was not confirmed. A possible explanation could be related to the fact that the fear stimulus was to aversive to promote any approach behaviors. Moreover, showing fewer behaviors often could also serve an adaptation purpose, given that the stimulus was considered frightening by children and even dangerous. Most important, these results suggest that the development of emotion regulation strategies may follow different pathways when it comes to positive versus negative affect contexts (particularly fear), and that positive affect and frustration/anger regulation may share common aspects (in quantity of strategies mobilized, not quality), since both situations develop around desired stimuli.

Toddlers' emotional expressiveness was also significantly influenced by the emotion-eliciting context experienced by children. Independently of the mothers' behavior, children exhibited expressiveness (either negative or positive) significantly more often, during fear and frustration/anger episodes, particularly fear, and fewer during positive affect episodes. These results do not confirm our initial hypothesis, but suggest that children may use expressiveness as an important way to elicit mothers' behaviors during difficult and negative emotional contexts. Specifically, when their survival perception is threatened (fear) or when they do not have enough resources to solve a situation (frustration/anger). In fact, recent studies have suggested that expressions of fear and anger may have co-evolved to mimic faces in order to enhance their communicative signal (Sacco \& Hugenberg, 2009). Most important, there seems to be a difference in the way children use behavioral strategies and emotional expressiveness as regulatory resources, according to the emotional context experienced (positive versus negative). Toddlers seem to express emotions more frequently during negative affect episodes (particularly fear), than during positive affect contexts, as a way to signal their mothers their distress. However, fewer behavioral strategies are exhibited during fear episodes, than during positive affect or frustration/anger episodes.

\subsection{Effects of maternal involvement on toddlers' emotion regulation}

Toddlers' behavioral strategies varied as function of maternal involvement, a result supported by previous studies (Diener \& Mangelsdorf, 1999a). Nevertheless, mothers' involvement seemed to influence the increase or decrease of certain strategies in detriment of others, according to the emotional context experienced. During fear episodes, when maternal involvement was observed children played with stimulus and approached it more often, than when mother constrained their behaviors (e.g., Diener \& Mangelsdorf, 1999a, 1999b; Grolnick et al., 1996). In frustration/anger episodes, maternal involvement was significantly associated with fewer fussing to mother, passive disengagement of attention, distraction and tension release behaviors show by children, when compared to mothers' constraint. During positive affect episodes, mothers' active participation also influenced children's behavior, either by increasing (engaging to mother strategies) or by reducing the frequency of their strategies (passive disengagement of attention, self-soothing, and stranger).

These findings suggest that different emotional contexts have different goals and therefore, different strategies should be used by children, namely, through the mothers' involvement. These results are consistent with the functionalist perspective of emotion regulation (Campos et al., 1994). As predicted, children showed behavioral strategies significantly more often when mothers were involved, and not constrained, during fear episodes as a way to achieve their context-specific goals (Campos et al., 1994), that is, to get comfort or to explore the toy safely. During positive affect episodes, no differences between mother constrained and involved periods were found, as expected. However, during frustration/anger episodes, toddlers did not exhibit more strategies during mother constrained periods, than during involved ones (as a way to get the toy back and play), as expected.

Children's emotional expressiveness did not vary as function of maternal involvement, but significant differences were found as a result of an interaction between context and maternal involvement. As supported by previous studies (Diener \& Mangelsdorf, 1999a; Gross \& Thompson, 2007; Thompson, 1994), our results indicated that children showed positive affect expressions significantly more often when the mothers became involved, either during frustration/anger or positive affect 
episodes This finding is particularly interesting, since both episodes developed around children's desire and approach behaviors towards the stimulus, particularly during frustration episodes, where the object was desirable, but unattainable. On the opposite, the mothers' involvement seemed to have had no significant impact on children's positive expressions during fear episodes, probably because the stimulus caused too much withdrawal reactions and emotions on children. When it comes to negative expressions (fear and frustration/anger), the mothers' involvement seemed to have a differential impact, according to the emotional context experienced. During fear episodes, it seemed to buffer children's negative affect, since negative affect expressions showed no significant differences from the constrained to the involved period. During frustration/anger episodes, mothers' involvement was significantly associated to lower levels of stress and frustration expressed by children. During positive affect episodes, children showed fewer fearful expressions when the mother was engaged, when compared to mother constrained periods. However, children expressed frustration/anger expressions significantly more often during mother involved periods, than during constrained ones, probably, due to the new negotiation behaviors that the mother's involvement created and imposed on children when it comes to sharing the desired positive affect stimulus during play. In fact, Van Kleef \& De Drue (2010) reported a relationship between negotiation behaviors and anger expression.

Overall, children did not exhibit significant differences in emotional intensity as a function of maternal involvement; they were only registered when the emotion-eliciting contexts were taken into account. This indicates the importance of interactions between mothers' behavior and emotion-eliciting context in the study of emotional intensity during the toddler's years.

On the contrary to results previously reported in the literature, focusing on toddlers' emotion regulation strategies during challenging contexts (see Buss \& Goldsmith, 1998; Diener \& Mangelsdorf, 1999a; Parritz, 1996), this work was developed at the children's homes and not at the laboratory. Nonetheless, all the stimuli used at the dyads' homes elicited the emotions they were designed to evoke, which means that emotion-eliciting context and maternal involvement manipulations can be induced both at controlled or naturalistic settings. Moreover, these results suggest that toddlers' emotion regulatory skills may be independent of children's familiarity perception towards the setting or place, where behaviors occur.

\subsection{Limitations and future research}

This study revealed some limitations, similar to Diener and Mangelsdorf's work (1999a), the mother constrained and involved periods were not counterbalanced, since during pilot testing, maternal involvement seemed to change the children's emotional interpretation of the stimuli, particularly, during fear episodes. In future research it would be very important to study mothers' characteristics (depression, anxiety, marital quality, etc.), which may lead to differences in the quality of the mothers' involvement.

\section{Acknowledgments}

The authors wish to thank all the children, families and institutions who participate in this study. This work was supported in part by grants from FCT to I \& D Unit No. 332/94 and SFRH/BD/23365/2005. We are also grateful to all the colleagues from Line 1 of UIPCDE - Developmental Psychology for their valuable comments.

\section{References}

Beauregard, M., Lévesque, J., \& Bourgouin, P. (2001). Neural correlates of the conscious self-regulation of emotion. Journal of Neuroscience, 21, 1-6. RC165. Bowlby, J. (1982). Attachment and loss: Vol. 1. Attachment. London: Hogarth Press. (Original work published in 1969).

Bretherton, I. (1990). Open communication and internal working models: Their role in the development of attachment relationships. In R. A. Thompson (Ed.), Socioemotional development. Lincoln: University of Nebraska Press.

Buss, K. A., \& Goldsmith, H. H. (1998). Fear and anger regulation in infancy: Effects on the temporal dynamics of affective expression. Child Development, 69, 359-374

Calkins, S. D. (1994). Origins and outcomes of individual differences in emotion regulation. In N. Fox (Ed.), The development of emotion regulation: Biological and behavioral considerations (pp. 53-69). Monographs of the Society for Research on Child Development, 59(2-3) (Serial N 240).

Calkins, S. D., \& Johnson, M. C. (1998). Toddler regulation of distress to frustrating events: Temperamental and maternal correlates. Infant Behavior and Development, 21, 379-395.

Campos, J. J., Mumme, D. L., Kermoian, R. K., \& Campos, R. G. (1994). A functionalist perspective on the nature of emotion. In N. Fox (Ed.), The development of emotion regulation: Biological and behavioral considerations (pp. 284-303). Monographs of the Society for Research on Child Development, 59(2-3) (Serial $\left.\mathrm{N}^{\circ} 240\right)$.

Cassidy, J. (1994). Emotion regulation: Influences of attachment relationships. In N. Fox (Ed.), The development of emotion regulation: Biological and behavioral considerations (pp. 229-249). Monographs of the Society for Research on Child Development Monograph, 59 (Serial N $\left.{ }^{\circ} 240\right)$.

Cole, P., Michel, M., \& Teti, L. (1994). The development of emotion regulation and dysregulation: A clinical perspective. In N. Fox (Ed.), The development of emotion regulation: Biological and behavioral considerations (pp. 73-100). Monographs of the Society for Research on Child Development, 59(2-3) (Serial $\mathrm{N}^{\circ} 240$ ).

Denham, S. A. (1998). Emotional development in young children. New York, NY: Guilford Press.

Diener, E., Sandvik, E., \& Pavot, W. (1991). Happiness is the frequency, not the intensity, of positive versus negative affect. In F. Strack, M. Argyle, \& N. Schwarz (Eds.), Subjective well-being - An interdisciplinary perspective. Pergamon Press.

Diener, M. L., \& Mangelsdorf, S. C. (1999). Behavioral strategies for emotion regulation in toddlers: Associations with maternal involvement and emotional expressions. Infant Behavior and Development, 22(4), 569-583.

Diener, M. L., \& Mangelsdorf, S. C. (1999b). Emotion regulation coding. Unpublished manuscript.

Fox, N. A. (1994). Dynamic cerebral processes underlying emotion regulation. In N. A. Fox (Ed.), The development of emotion regulation: Biological and behavioral considerations (pp. 152-166). Monographs of the Society for Research in Child Development, 59(2-3) (Serial $\left.\mathrm{N}^{\circ} 240\right)$. 
Gilliom, M., Shaw, D. S., Beck, J. E., Schonberg, M. A., \& Lukon, J. L. (2002). Anger regulation in disadvantaged preschool boys: Strategies, antecedents, and the development of self-control. Developmental Psychology, 38(2), 222-235.

Giuliani, N. R., McRae, K., \& Gross, J. J. (2008). The up- and down-regulation of amusement: Experiential, behavioural and autonomic consequences. Emotion, $8(5), 714-719$.

Grolnick, W. S., Bridges, L. J., \& Connell, J. P. (1996). Emotion regulation in two year-olds: Strategies and emotional expression in four contexts. Child Development, 67, 928-941.

Gross, J. J., \& Thompson, R. A. (2007). Emotion regulation: Conceptual foundations. In J. J. Gross (Ed.), Handbook of emotion regulation (pp. 3-26). New York: Guilford Press.

Kim, S. H., \& Hamann, S. (2007). Neural correlates of positive and negative emotion regulation. Journal of Cognitive Neuroscience, 19(5), 776-798.

Kopp, C. B. (1989). Regulation of distress and negative emotions: A developmental view. Developmental Psychology, 25(3), $343-354$.

Malott, R. W., \& Trojan, E. A. (2008). Principles of behavior (6th edition). Upper Saddle River, NJ: Prentice Hall.

Maughan, A., \& Cicchetti, D. (2002). Impact of child maltreatment and interadult violence on children's emotion regulation abilities and socioemotional adjustment. Child Development, 73, 1525-1542.

Nachmias, M., Gunnar, M., Mangelsdorf, S., Parritz, R. H., \& Buss, K. (1996). Behavioral inhibition and stress reactivity: The moderating role of attachment security. Child Development, 67, 508-522.

Parritz, R. H. (1996). A descriptive analysis of toddler coping in challenging circumstances. Infant Behavior and Development, 19(2), 171-180.

Rothbart, M. K., \& Sheese, B. E. (2007). Temperament and emotion regulation. In J. J. Gross (Ed.), Handbook of emotion regulation (pp. 3-26). New York: Guilford Press.

Sacco, D. F., \& Hugenberg, K. (2009). The look of fear and anger: Facial maturity modulates recognition of fearful and angry expressions. Emotion, 9(1), 39-49.

Thompson, R. A. (1994). Emotion regulation: A theme in search of definition. In N. A. Fox (Ed.), The development of emotion regulation: Biological and behavioral considerations (pp. 25-52). Monographs for the Society for Research on Child Development, 59(2-3) (Serial N $\left.{ }^{\circ} 240\right)$.

Thompson, R. A., \& Meyer, S. (2007). Socialization of emotion regulation in the family. In J. J. Gross (Ed.), Handbook of emotion regulation (pp. 3-26). New York: Guilford Press.

Tugade, M. M., \& Fredrickson, B. L. (2007). Regulation of positive emotions: Emotion regulation strategies that promote resilience. Journal of Happiness Studies, 8(3), 311-333.

Van Kleef, G. A., \& De Drue, C. K. W. (2010). Long-term consequences of anger expression in negotiation: Retaliation or spillover? Journal of Experimental Social Psychology, 46(5), 753-760. 